\title{
Optimal Wind Turbines Micrositing in Onshore Wind Farms Using Fuzzy Genetic Algorithm
}

\author{
Jun Yang, Rui Zhang, Qiuye Sun, and Huaguang Zhang \\ College of Information Science and Engineering, Northeastern University, Shenyang 110819, China \\ Correspondence should be addressed to Jun Yang; yangjun@mail.neu.edu.cn
}

Received 17 October 2014; Accepted 15 February 2015

Academic Editor: Victor Sreeram

Copyright (C) 2015 Jun Yang et al. This is an open access article distributed under the Creative Commons Attribution License, which permits unrestricted use, distribution, and reproduction in any medium, provided the original work is properly cited.

\begin{abstract}
With the fast growth in the number and size of installed wind farms (WFs) around the world, optimal wind turbines (WTs) micrositing has become a challenge from both technological and mathematical points of view. An appropriate layout of wind turbines is crucial to obtain adequate performance with respect to the development and operation of the wind power plant during its life span. This work presents a fuzzy genetic algorithm (FGA) for maximizing the economic profitability of the project. The algorithm considers a new WF model including several important factors to the design of the layout. The model consists of wake loss, terrain effect, and economic benefits, which can be calculated by locations of wind turbines. The results demonstrate that the algorithm performs better than genetic algorithm, in terms of maximum values of net annual value of wind power plants and computational burden.
\end{abstract}

\section{Introduction}

Nowadays, wind energy plays a very important role in the field of renewable energy supply worldwide. According to Global Wind Energy Council (GWEC), the new global total wind power capacity was $318,105 \mathrm{MW}$ by the end of 2013, representing more than 12.5 percent increase in cumulative market [1]. Wind power has become the most valuable and promising renewable energy option not only in developed but also in developing countries [2]. Wind energy technology develops rapidly and its cost is beginning to actually compete with existing fossil-fuel power production methods. But with the fast growth in the number and size of installed wind farms around the world, optimal wind turbines micrositing has become very important topic of study. The optimal turbines layout of a wind farm is a challenge from both mathematical and technological points of view. An appropriate position of wind turbines will directly influence the costs and the produced energy of a wind farm. The generated power from the turbines of a wind farm is often lower than expected, partly because the aerogenerators receive lower wind speeds and less energy captures if they are located behind the other one. This effect is called the wake effect [3].
So far, several works have appeared in which the optimization of a mathematical model of a wind farm has been addressed in order to undertake the optimal position of WTs [4-6]. In these studies, the optimized objective functions taking into account maximum energy production and minimum levelized cost of energy were used for determining the optimal locations of WTs. The problem was introduced by Mosetti et al. [7] which aimed to maximize the annual energy produced (AEP) and to minimize the installation costs by assuming a rather simplified cost model of the wind farm (based on economies of scale and the overlapping of wakes) to search for an optimal layout based on genetic algorithms (GAs). And the algorithm used a wake effect analysis similar to that of Katic et al. [8]. The following methods have considered Monte Carlo simulation with a simple model [6, 9], ant colony optimization (ACO) algorithm requiring large amount of calculation [10]. Geem and Hong [11] optimize the layout with the constraints of resources or budget bounds by an improved formulation method, but the cost and power functions are also experience function and cannot reflect the real WF model very well. Some other WT micrositing optimization methods, based on the Gaussian particle swarm optimization with local search strategy [12], binary particle 
swarm optimization [5], and greedy algorithm [13], have also been studied.

In the current study, we establish a new model for WF considering the main effective indicators simultaneously, including wake loss, terrain effect, hub height of the turbines, and economic benefit. Then we present an efficient methodology for the optimization of onshore WFs. In our approach, fuzzy control is implemented to adjust the crossover probability and mutation probability of the GA for determining the best sites of WTs in two different scenarios.

After this introduction, the paper is organized as follows. Section 2 presents a WF model including wake model, terrain effect model, energy production model, and economic model. Section 3 describes the approach to the problem and the proposed methodology. Test cases are provided in Section 4. At last, conclusions are discussed in Section 5.

\section{Wind Farm Modelling}

2.1. Wake Effect Model. The analytical model in this paper is similar to the wake decay model proposed by Jensen [14]. Depending on the wind farm geometry, the wind speed decay ratio $C_{w}$ produced in the airflow when the wind passes through the rotor of a wind turbine (see Figure 1) can be calculated by the expression:

$$
C_{w}=\frac{\left(u_{x}-u_{0}\right)}{u_{0}}=-\frac{2 a}{(1+2 k X / D)^{2}},
$$

where $u_{0}$ is the initial free stream velocity, $u_{x}$ is the velocity in the wake at a distance $X$ downstream of the upwind turbine, $D$ is the diameter of the upwind turbine, and $k$ is the wake decay constant. $a$ is the axial induction factor or inflow factor that is related to the thrust coefficient, $C_{T}$, according to

$$
C_{T}=4 a(1-a) .
$$

The expansion rate $k$, which is known as wake decay constant, is a value of 0.075 for onshore cases and 0.05 for offshore ones suggested in the literature [15] and is calculated as a function of surface roughness, given as

$$
k=\frac{0.5}{\ln \left(z / z_{0}\right)},
$$

where $z$ is the hub height of the wind turbine and $z_{0}$ is surface roughness.

2.2. Terrain Effect Model. The terrain of onshore wind farm may be complex. It is necessary to consider topographic influence on the behavior of the wind. The effect of topography on surface layer wind is very big. To estimate the wind speed $U_{p}$, at a prediction height, $Z$, the guidelines in [16] can be used. And the wind speed changes (see Figure 2) caused by both the topographic feature and a step change in surface roughness are considered. According to Weng et al. [16], the wind speed change $\Delta S(0, Z)$ can be calculated as follows:

$$
\frac{\Delta S(0, Z)}{\Delta S_{\max }}=\exp \left\{A_{1}-A_{2}\left(\frac{Z}{L_{1}}\right)-A_{1} e^{-A_{3}\left(Z / L_{1}\right)}\right\}
$$

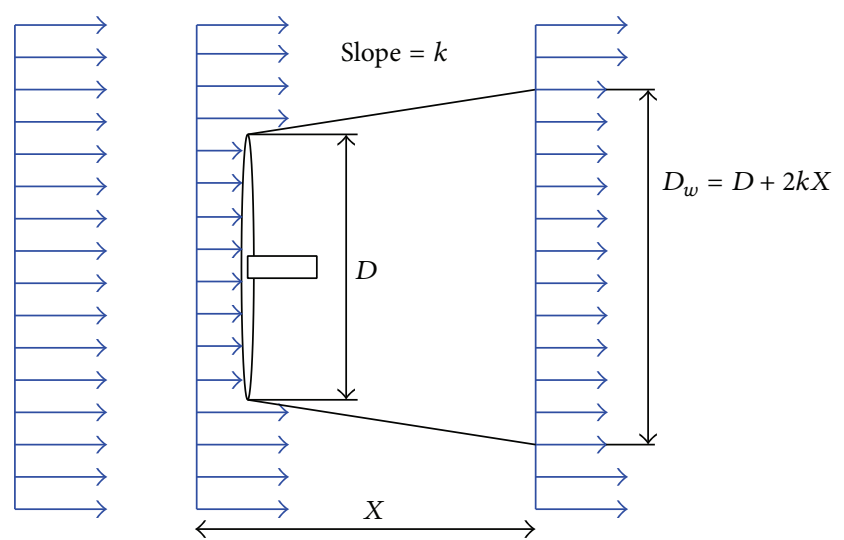

FIgURE 1: Schematic representation of the wind speed field in the wake.

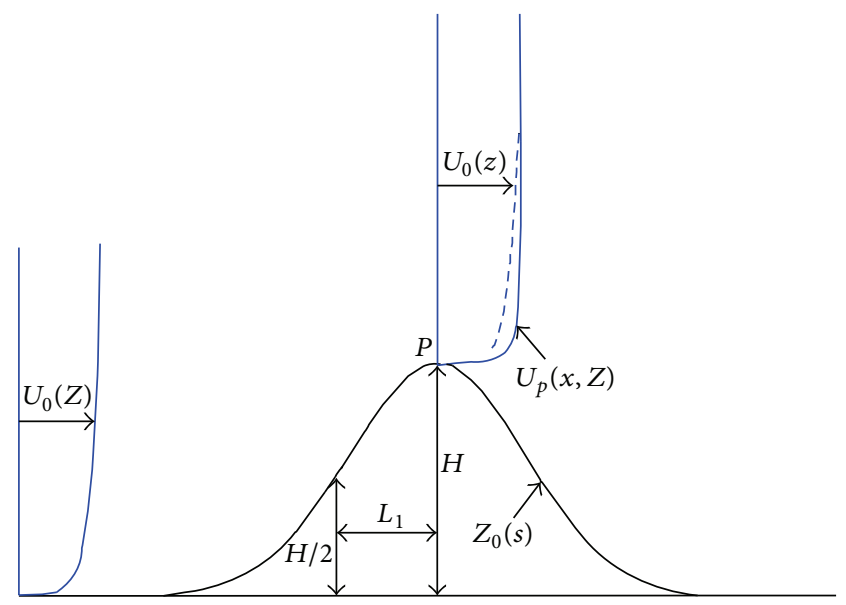

FIGURE 2: Schematic diagram of airflow over complex terrain situation.

where $\Delta S_{\max }$ is the maximum fractional speedup ratio at the hilltop, according to (5). $A_{i}(i=1,2,3)$ are constants to be determined. $L_{1}$ is a horizontal length scale defined as the distance from the hilltop or the bottom of the valley to the upstream point where the elevation is $0.5 \mathrm{H}$ :

$$
\begin{aligned}
& \Delta S_{\max } \\
& \quad=\frac{H}{L_{1}}\left(B_{1}+B_{2}\left(\frac{L_{1}}{z_{0}}\right)\right)\left(1+B_{3}\left(\frac{H}{L_{1}}\right)+B_{4}\left(\frac{H}{L_{1}}\right)^{2}\right),
\end{aligned}
$$

where $B_{i}(i=1,2,3,4)$ are constants listed in Table 1 and $z_{0}$ is surface roughness value.

2.3. Energy Production Model. The typical characteristic of wind speed behavior is a Weibull distribution [17]. The probability (frequency or percentage of time) of wind speed is defined by the parameters of scale, $C$, and shape, $K$, as shown in

$$
f(u)=\frac{K}{C} \cdot\left(\frac{u}{C}\right)^{K-1} \cdot e^{-(u / C)^{K}} .
$$


TABLE 1: Constant $B_{i}$ for $2 \mathrm{D}$ and 3D rolling terrain and isolated hills [16].

\begin{tabular}{lcccc}
\hline Constant & $B_{1}$ & $B_{2}$ & $B_{3}$ & $B_{4}$ \\
\hline 2D rolling terrain & 2.20 & -0.049 & -0.64 & -0.19 \\
2D isolated hill & 2.40 & -0.051 & 0.029 & -0.51 \\
3D rolling terrain & 1.58 & -0.036 & 0.069 & -0.85 \\
3D isolated hill & 2.05 & -0.048 & 0.24 & -0.40 \\
2D valley & 2.57 & -0.054 & -0.65 & -0.80 \\
\hline
\end{tabular}

Compared to WF selected in flat area, both wake effect and terrain effect should be taken into account in complex terrain. The wind speed on the aerogenerator $u_{x}$ can be calculated as

$$
u_{x}=\left(1+C_{w}\right)\left(u_{0}+C_{o} \cdot \Delta S\right) \text {, }
$$

where $C_{w}$ is wind speed change ratio caused by wake and can be calculated by (1), while $\Delta S$ is wind speedup induced by orography and can be calculated by (4). $C_{o}$ can be set to zero if the WF is located in flat area; otherwise, it should be taken the value of 1 . Finally, the AEP of a WF is calculated:

$$
\mathrm{AEP}_{\mathrm{WF}}=\sum_{i=1}^{N} T_{i} \int_{u_{\mathrm{CI}_{i}}}^{u_{\mathrm{CO}_{i}}} P_{\mathrm{WT}_{i}}\left(u_{x i}\right) f\left(u_{x i}\right) d u,
$$

where $T_{i}$ is the number of the $i$ th WT available hours per year (h), $N$ is the number of WTs, and $u_{\mathrm{CI}_{i}}$ and $u_{\mathrm{CO}_{i}}$ are the cutin speed and cut-out speed, respectively. $P_{\mathrm{WT}_{i}}$ is the power curve of the $i$ th WT and $u_{x i}$ is the $i$ th WT's actual wind speed taking wake effect and terrain effect into account, which can be calculated by (7).

2.4. Economic Model. Usually in the investment project economic analysis, net annual value (NAV) has been used as a measure of economic benefits of workflow project. The NAV of a WF is calculated by the following expression:

$$
\begin{aligned}
\operatorname{NAV}(t)= & -\frac{E_{c}(t) \cdot(1+r)^{n} \cdot r}{(1+r)^{n}-1} \\
& -E_{s}(t)+E_{\text {en }}(t)+\operatorname{AEP}(t) \times p,
\end{aligned}
$$

where $t$ is WT life span, $r$ is the basic discount rate, $p$ is the sale price per $\mathrm{kWh}, \operatorname{AEP}(t)$ is WF annual energy produced calculated by (8). If we consider the energy-saving effect of wind power generation, energy-saving emission reduction contribution of $E_{\text {en }}(t)$ should be offset by the same generation. The WF initial construction investment costs, $E_{c}$, and the WF operation and maintenance costs, $E_{s}$, are two main aspects of the investment cost, and they can be computed as

$$
E_{c}(t)=E_{c p}(t)+E_{c e}(t)+E_{c c}(t)+E_{c i}(t)+E_{c o}(t),
$$

where $E_{c}(t)$ is divided into four main parts: WTs purchase cost, $E_{c p}(t)$; WF electrical infrastructure cost, $E_{c e}(t)$; civil work, $E_{c c}(t)$; installation and transport cost, $E_{c i}(t)$; and others, $E_{c o}(t)$ :

$$
E_{s}(t)=\left(E_{s c}(t)+E_{s s}(t)+E_{s n}(t)+E_{s t}(t)\right) \cdot t,
$$

TABLE 2: Typical construction cost breakdown of an onshore WF.

\begin{tabular}{lc}
\hline WTs (\%) & $65-75$ \\
Electrical infrastructure (\%) & $1-10$ \\
Collector system & $6-9$ \\
Transmission system & $2-3$ \\
Substation & $2-3$ \\
Civil work (\%) & $0-5$ \\
Installation and transport (\%) & $0-2$ \\
Others (\%) & 5 \\
\hline
\end{tabular}

where $E_{s}(t)$ has been broken down into annual depreciation charge, $E_{s c}(t)$; annual operation and annual maintenance cost, $E_{s s}(t)$; annual additional cost for connecting in parallel with system, $E_{s n}(t)$; and annual tax, $E_{s t}(t)$.

Table 2 shows the typical schedule of onshore WF construction cost. As shown, most of the WF construction costs are associated with the acquisition of WTs. The cost of foundation and electrical infrastructure also takes an important role. However, the proportion of each factor will depend on the specific characteristics of the WF studied.

\section{Proposed Methodology}

As previously mentioned, several heuristic optimization techniques have been used to solve the best WT microsite in WF problems. In particular, the most widely used optimization method is that of GAs. But GAs often lead to some phenomena such as slow convergent speed and premature convergence [18]. In order to solve this problem, fuzzy genetic algorithm (FGA) is proposed in this work to adjust the values of crossover probability $P_{c}$ and mutation probability $P_{m}$ adaptively so as to optimize the genetic algorithm. The flowchart of the FGA implemented is depicted in Figure 3.

3.1. Initialization of the Population. The process begins with creating the initial population, which is composed of a group of randomly generated individuals (potential solutions). The chromosomes are encoded in the real valued turbine coordinates, which are not beyond the WF area boundaries limit and the composition of each individual chromosome is given by

$$
X=\left[\begin{array}{lllllll}
x_{1} & y_{1} & x_{2} & y_{2} & \cdots & x_{k} & y_{k}
\end{array}\right],
$$

where $\left(x_{1}, y_{1}\right)$ and $\left(x_{k}, y_{k}\right)$ correspond to the coordinates of the first and $k$ th WTs, respectively. Set the iterative time $t=0$ and the maximal iterative time as $T$.

3.2. Individual Estimation. Calculate the individual's fitness of the population which is expressed as

$$
f= \begin{cases}\operatorname{NAV}(t)+C_{\text {min }} & \text { if } \operatorname{NAV}(t)+C_{\text {min }}>0 \\ 0 & \text { if } \operatorname{NAV}(t)+C_{\min } \leq 0,\end{cases}
$$

where NAV $(t)$ is the NAV of WFs, according to (9). $C_{\text {min }}$ is an appropriate relatively small number, and it can be got by a prespecified smaller number. 


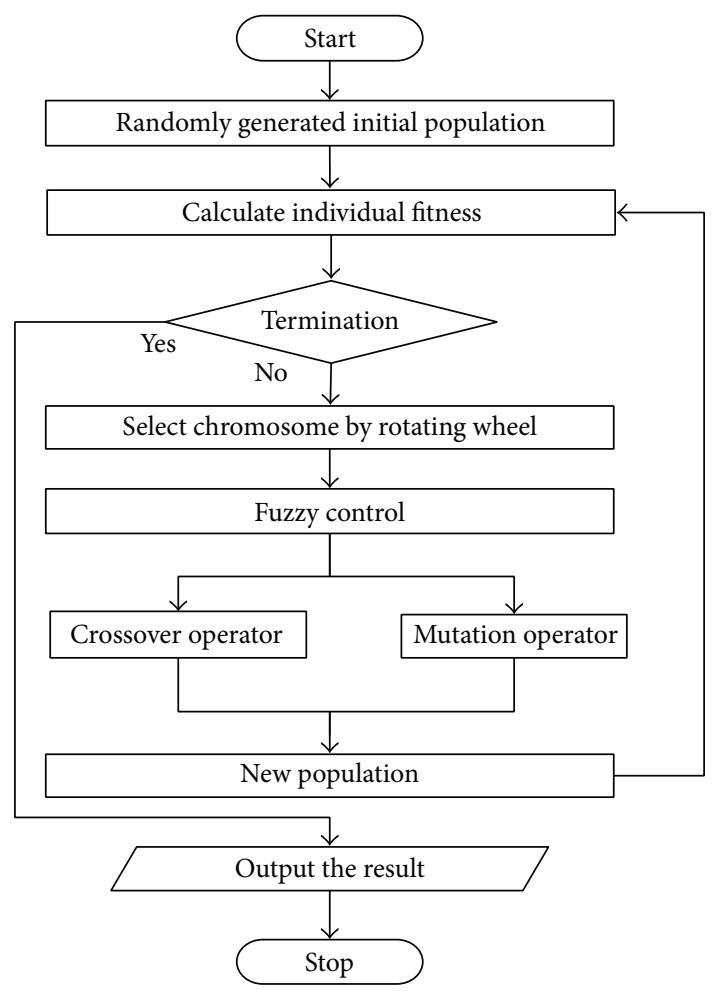

FIGURE 3: Block diagram of the proposed FGA method.

3.3. Constraints and Termination Criterion. Besides the turbines have to be placed inside the WF area, a minimum distance of five turbine rotor diameters $(5 D)$ between neighboring turbines was considered for obtaining feasible results for the optimal WT micrositing problem, and the constraints are expressed as

$$
\left(x_{i}-x_{j}\right)^{2}+\left(y_{i}-y_{j}\right)^{2} \geq 25 D^{2}
$$

where $\left(x_{i}, y_{i}\right)$ and $\left(x_{j}, y_{j}\right)$ are the coordinates of neighboring turbines. A user specified maximum number of generations were set as the termination criterion.

3.4. Selection Operation. The FGA goes on by selecting some solutions from the population to create a mating pool if the termination criterion is not satisfied. To populate the mating pool, roulette wheel selection is employed to first calculate each individual $X$ 's corresponding proportion of its fitness value to the total fitness value of the whole population, labeled as $p_{i}$, by

$$
p_{i}=\frac{f\left(X_{i}\right)}{\sum_{j=1}^{N} f(X)_{j}}
$$

where $i=1,2, \ldots, N$ and $N$ is the size of population. Then the operator repeats $N$ times of selecting an individual from the current population to generate the new population. In each time, a random real number uniformly scattered in the range
$[0,1)$ is generated, and the individual $k_{X}$ where $k$ satisfies $(16)$ is selected:

$$
k=\min \left\{j \mid \sum_{i=1}^{j-1} p_{i} \leq q, j=1,2, \ldots, N\right\} .
$$

3.5. Fuzzy Control. The fuzzy logic method is the reasonable candidate method for dynamically adjusting the crossover probability and mutation probability, the GA, because it is appropriate for representing uncertain knowledge [19-23]. A two-dimension fuzzy control is used in our FGA. There are two parameters $e_{1}$ and $e_{2}$ as inputs of fuzzy inference system. The two parameters denoting the crossover and mutation probabilities, respectively, are subject to the fuzzy inference system and describe the excellence degree of the individual to cross and mutate. They are expressed as

$$
\begin{gathered}
e_{1}(t)=\frac{f_{\max }(t)-f_{\max }(t-1)}{f_{\text {max }}(t)-f_{\text {ave }}(t)}, \\
e_{2}(t)=\frac{f_{\text {ave }}(t)-f_{\text {ave }}(t-1)}{f_{\text {max }}(t)-f_{\text {ave }}(t)},
\end{gathered}
$$

where $t$ is the evolution generation, $f_{\text {ave }}(t)$ is the average population fitness at generation $t, f_{\max }(t)$ is the maximum population fitness at generation $t$, and $f_{\text {ave }}(t-1)$ is the average population fitness at the previous generation of $t . e_{1}$ and $e_{2}$ will be quantified as the input of the fuzzy controller, $E_{1}$ and $E_{2}$, by quantification factors. $E_{1}$ and $E_{2}$ corresponding to $e_{1}$ and $e_{2}$ are expressed as

$$
\begin{aligned}
& E_{1}=\left\langle\frac{2 n_{1}}{\left(b_{1}-a_{1}\right)} \cdot\left[e_{1}-\frac{\left(a_{1}+b_{1}\right)}{2}\right]\right\rangle, \\
& E_{2}=\left\langle\frac{2 n_{2}}{\left(b_{2}-a_{2}\right)} \cdot\left[e_{2}-\frac{\left(a_{2}+b_{2}\right)}{2}\right]\right\rangle,
\end{aligned}
$$

where " \langle\rangle " represents rounding to the nearest integer arithmetic. $\left[a_{1}, b_{1}\right]$ is the range of $e_{1}$, and $\left[a_{2}, b_{2}\right]$ is the range of $e_{2}$. [ $\left.-n_{1}, n_{1}\right],\left[-n_{2}, n_{2}\right]$ are the domain of discourse of $e_{1}, e_{2}$ respectively; then the definition of $E_{1}$ and $E_{2}$ word sets is expressed as

$$
\begin{gathered}
T\left(E_{1}\right)=\{\mathrm{ZO}, \mathrm{PS}, \mathrm{PB}\}, \\
T\left(E_{2}\right)=\{\mathrm{NB}, \mathrm{NS}, \mathrm{ZO}, \mathrm{PS}, \mathrm{PB}\},
\end{gathered}
$$

where NB is negative big, NS is negative small, ZO is zero, PS is positive small, and $\mathrm{PB}$ is positive big.

The current fuzzy input fuzzy controller can be obtained by $E_{1}$ and $E_{2}$ corresponding to maximum fuzzy membership value.

There is one important idea in the genetic algorithm determining the values of $P_{c}$ and $P_{m}$. When the difference between $f_{\max }(t)$ and $f_{\max }(t-1)$ varies widely, it means the current population in the normal evolution stage, $P_{c}$ and $P_{m}$, takes desirable normal. When $f_{\max }(t)$ and $f_{\max }(t-1)$ are very close, which can be thought of as premature convergence, $P_{c}$ should be greatly reduced and $P_{m}$ should be increased 
TABLE 3: Fuzzy rules of $\Delta P_{c}(t)$ for crossover operation.

\begin{tabular}{cccccc}
\hline$U_{1}$ & & & $E_{2}$ & & \\
& NB & NS & ZO & PS & PB \\
\hline$E_{1}$ & & & & & \\
ZO & NS & NB & NB & NB & NB \\
PS & ZO & ZO & NB & ZO & ZO \\
PB & NS & ZO & NS & PS & PB \\
\hline
\end{tabular}

TABLE 4: Fuzzy rules of $\Delta P_{m}(t)$ for mutation operation.

\begin{tabular}{cccccc}
\hline$U_{2}$ & & & $E_{2}$ & & \\
& NB & NS & ZO & PS & PB \\
\hline$E_{1}$ & & & & & \\
ZO & PS & PB & PB & PB & PS \\
PS & ZO & ZO & PB & ZO & NS \\
PB & PS & ZO & PS & NS & NB \\
\hline
\end{tabular}

for big mutation operation. When $f_{\text {ave }}(t)$ and $f_{\text {ave }}(t-1)$ are very close, it means these two generations are similar in population structure, so $P_{c}$ can be appropriately reduced and $P_{m}$ should be increased to increase the population structure of individuals. Based on the above described experience, Tables 3 and 4 ( $U_{1}$ and $U_{2}$ are controlled quantities) show the rule base used by the fuzzy operation presented. Then the actual controlled quantities acting on the controlled object are calculated as follows:

$$
\begin{aligned}
& \Delta P_{c}(t)=K_{u 1} \cdot U_{1}, \\
& \Delta P_{m}(t)=K_{u 2} \cdot U_{2},
\end{aligned}
$$

where $K_{u 1}$ and $K_{u 2}$ are the scaling factors.

The robust stability of the control system via a fuzzy estimator has been studied in [24]. Finally, we use the crisp value to modify the parameters $P_{c}$ and $P_{m}$ as follows:

$$
\begin{gathered}
P_{c}(t+1)=P_{c}(t)+\Delta P_{c}(t), \\
P_{m}(t+1)=P_{m}(t)+\Delta P_{m}(t) .
\end{gathered}
$$

3.6. Crossover and Mutation Operations. The probabilities of crossover and mutation, $P_{c}(t)$ and $P_{m}(t)$, are derived from the outputs of the fuzzy operation. After a new population is created, it is evaluated once again with regard to the fitness function. Besides, every individual must satisfy the requirements of the minimum distance between the WTs and other constraints. If the generated coordinates of WTs are not meeting the restrictions, then they will be cleared.

\section{Cases Study}

In order to determine the suitability of the presented methodology for the optimization problem of onshore WF, a set of study cases have been analyzed. The WF located in China is considered to have 10 identical turbines and an installed capacity of $15 \mathrm{MW}$. The WTs, used in this work, have a hub height of $72 \mathrm{~m}$ and a $41 \mathrm{~m}$ rotor radius. Figure 4 displays the power curve of a single $1.5 \mathrm{MW}$ wind turbine.

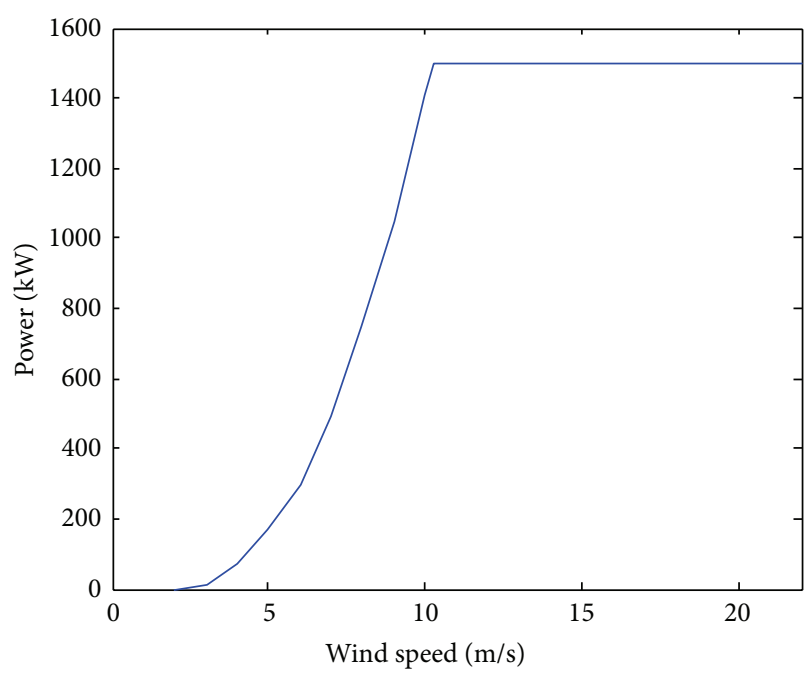

FIGURE 4: 1.5 MW turbine power curve.

In order to evaluate speedup caused by terrain and the wake losses, information regarding the wind is needed. In Figure 5, it is shown that the wind rose with the average wind speeds and respective frequencies that were used in this work and the wind power of the location of WT can be obtained by the siting of distributed WFs [25].

In order to compare the optimization strategies, the proposed approaches are optimized through the FGA for 100 generations. The WF is considered to have 20 years' life span and the basic discount rate is regarded as $8 \%$. The sale price is $¥$ (China Yuan) 0.50 per kWh. Simultaneously, the GA method is run. This approach is terminated once both proposed variants are completed. The results obtained with the optimization approaches are presented and discussed and a comparison between the results obtained with the optimization methods is performed as follows. The algorithm has been implemented by using the mix programming of C\#.net and MATLAB. Arcgis engine which is a collection of GIS components and developer resources that can be embedded, allowing us to add dynamic mapping and GIS capabilities to existing applications or build new custom mapping applications, is also applied to better deal with terrain effect. The method was performed on a computer which has an Intel Core 2 Duo E7500 @2.93 GHz processor with 2 GB RAM.

4.1. Case 1. In this case, we assume that the site of WF is selected in a plain area, and the effect of terrain on the wind can be ignored. So when calculating the wind speed on the aerogenerator, $C_{o}$ should be set to zero. Initially, the WTs are placed in a distance of about 500 meters between them. The wind resource distribution of the WF can be obtained according to Figure 5. In the cause of demonstrating the ability of the proposed methodology, Figure 6 presents the scenario of WTs layout with the change evaluations, and the points in the figure represent the locations of WTs. From the evolution, it is clear that the prevailing wind direction should 


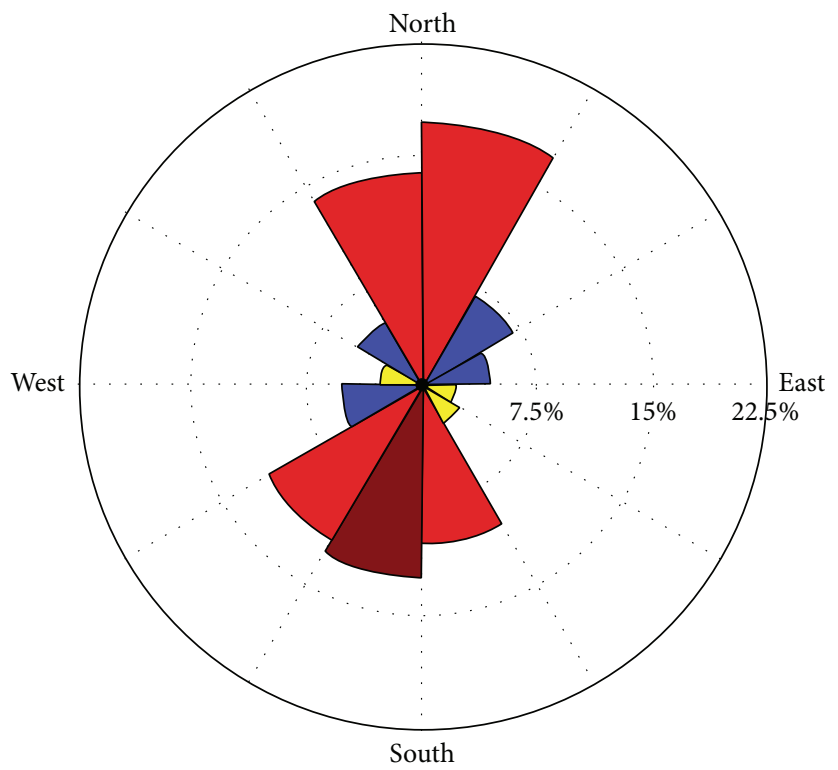

Wind speed
\begin{tabular}{|l}
$\square-5 \mathrm{~m} / \mathrm{s}$ \\
$4-7 \mathrm{~m} / \mathrm{s}$ \\
$5-6 \mathrm{~m} / \mathrm{s}$ \\
$\square-8 \mathrm{~m} / \mathrm{s}$
\end{tabular}

FIgURE 5: Wind rose with the frequencies and speed.

TABLE 5: Main results for the configurations of Case 2.

\begin{tabular}{lccc}
\hline & Initial & Final (FGA) & Final (GA) \\
\hline Average wake loss $(\%)$ of WTs & 0.16 & 0.07 & 0.06 \\
Average wind speedup (\%) of WTs due to terrain & 8.14 & 9.87 & 4.73 \\
WF AEP $(\mathrm{GWh})$ & 32.44 & 43.65 & 72.34 \\
WF construction cost $E_{c}(¥)$ & $7.80 \times 10^{8}$ & $7.50 \times 10^{8}$ & $7.62 \times 10^{8}$ \\
WF annual operation and maintenance costs $E_{s}(¥)$ & $3.50 \times 10^{5}$ & $3.30 \times 10^{5}$ & $3.37 \times 10^{5}$ \\
Energy-saving emission reduction contribution $E_{\text {en }}(¥)$ & $7.60 \times 10^{5}$ & $10.23 \times 10^{5}$ & $9.92 \times 10^{5}$ \\
WF NAV (¥) & $1.63 \times 10^{10}$ & $2.19 \times 10^{10}$ & $2.11 \times 10^{10}$ \\
\hline
\end{tabular}

be filled with more aerogenerators. As can be seen from Figure 6(d), FGA can be implemented with WTs layout like the GA. Compared with the initial layout, the final distance between WTs is more reasonable to reduce the influence of the wake.

4.2. Case 2. In this case, we assume that the WF is in complex terrain area. Both wake loss and terrain effect are considered. So when calculating the wind speed on the aerogenerator, $C_{o}$ should be set to 1 . The initial and final positions of each one of the WTs corresponding to the final configuration achieved by FGA (blue points) and GA (red points) in the complex terrain areas can be observed in Figures 7 and 8. It is possible to observe that the majority of WTs are located in the dominant wind direction. The results of WTs layout optimized by means of FGA and GA, respectively, are almost the same. In Table 5, the main results are compared, the wake losses are got by (1), and wind acceleration due to terrain effect is obtained according to (4). The NAV from the obtained WF configuration is obtained by means of (9). As can be seen from Table 5, after optimization using FGA, the wake losses of WF are reduced, wind acceleration due to terrain effect is improved, and the economic benefits of WF have been significantly improved. Comparing with GA, FGA performs better in improving the profits of the WF by an $8 \%$ increase in NAV (see Table 5).

In order to show the ability of the proposed methodology to achieve the optimal solution, Figure 9 shows the best fitness values during the optimizations using two methods. The fitness values will converge to the optimal value at about 12 generations using FGA, while using GA to optimize the layout will converge at 22 generations. So it is obvious that FGA has the advantage of converging to the global optimal with a faster speed than GA.

From the above cases, it proves that the mathematical modeling of WF is sufficient and suitable for optimization of the layout of WTs using the FGA. Furthermore, considering wider application, the modeling extension adding load data 


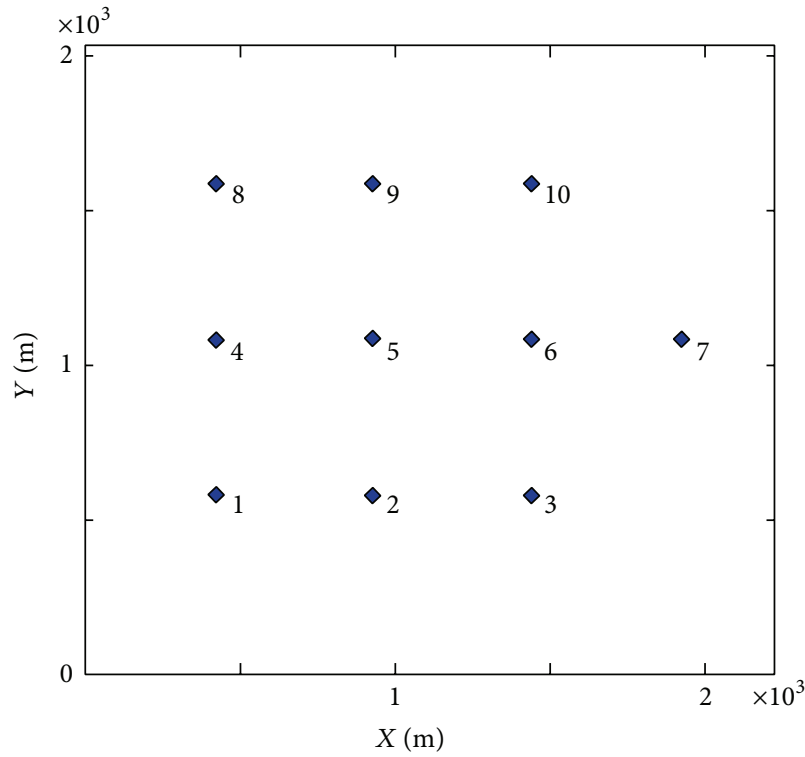

$\diamond \mathrm{FGA}$

(a) The scenario at 0 evaluations

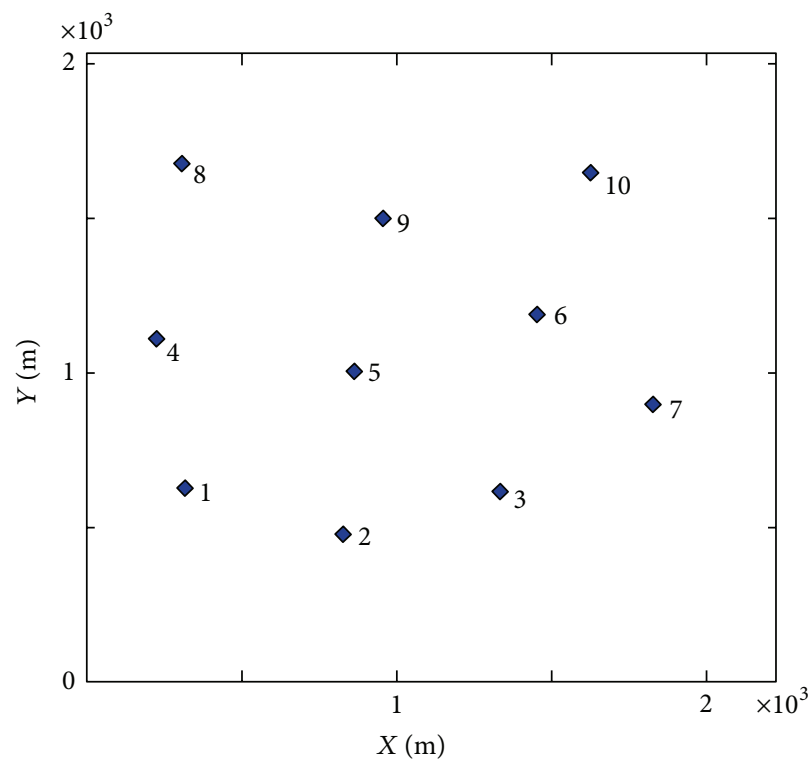

$\diamond$ FGA

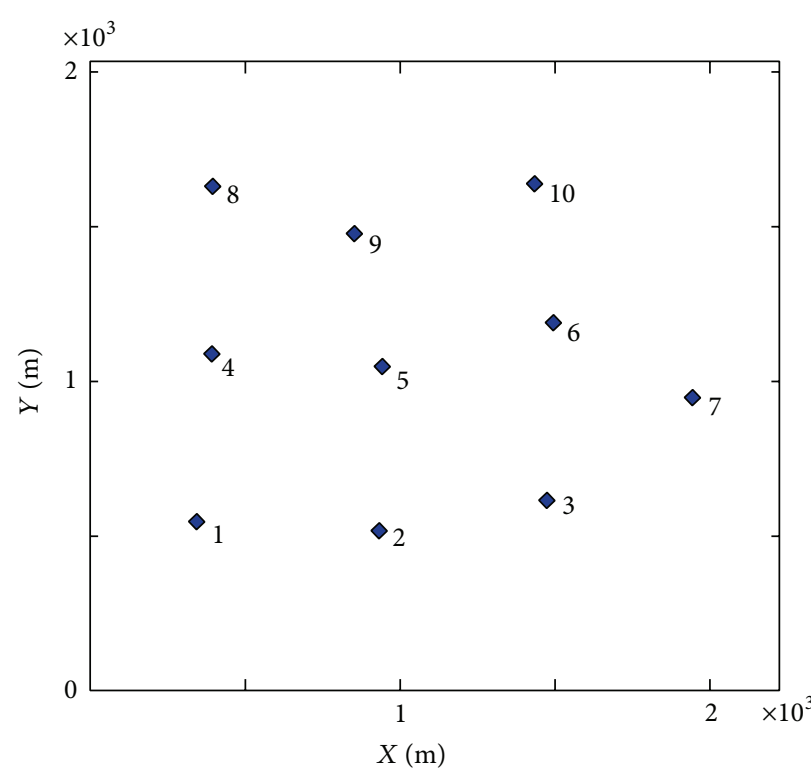

$\diamond$ FGA

(b) The scenario at 10 evaluations

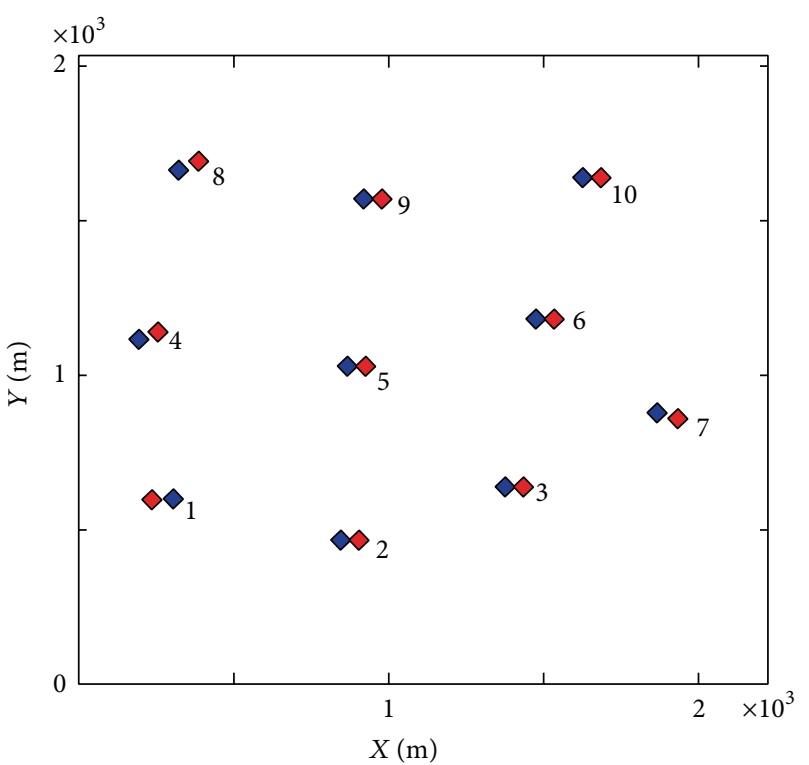

FGA

GA

(c) The scenario at 50 evaluations

(d) The scenario at 100 evaluations

FIgURE 6: The scenario of WTs layout with the change evaluations of Case 1.

and load modeling can also be used in the controllers for wind turbines and wind power prediction so as to realize coordinated control of reactive power of the whole WF.

\section{Conclusions}

For the purpose of addressing the problem of the optimal wind turbines micrositing in onshore wind farms, a complete model has been developed considering the main aspects involved in wind farm. The optimization method proposed in this paper, due to the crossover and mutation probabilities controlled by fuzzy rules, provides optimal WTs layout with lower computational cost. At last, the suitability and computational efficiency of the FGA have been successfully demonstrated by analyzing the micrositing of wind turbines problem in the case of onshore wind farms.

Although we considered one specific onshore WFs model, it is important to note that our optimization algorithm can also be easily applied to offshore WFs. 


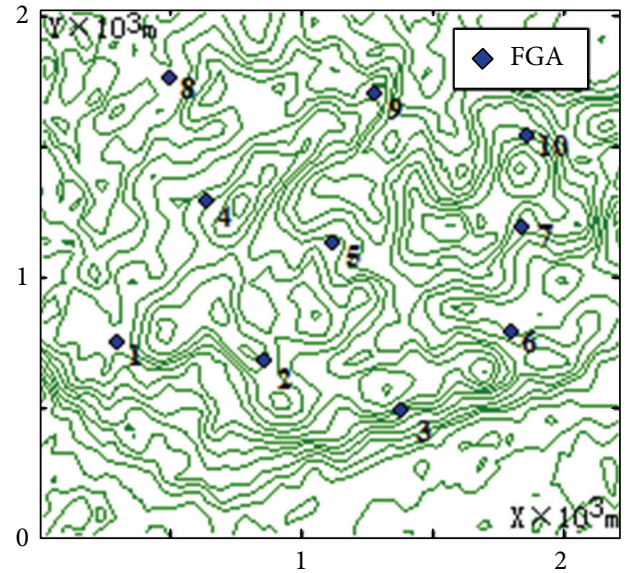

FIGURE 7: Initial WTs layout.

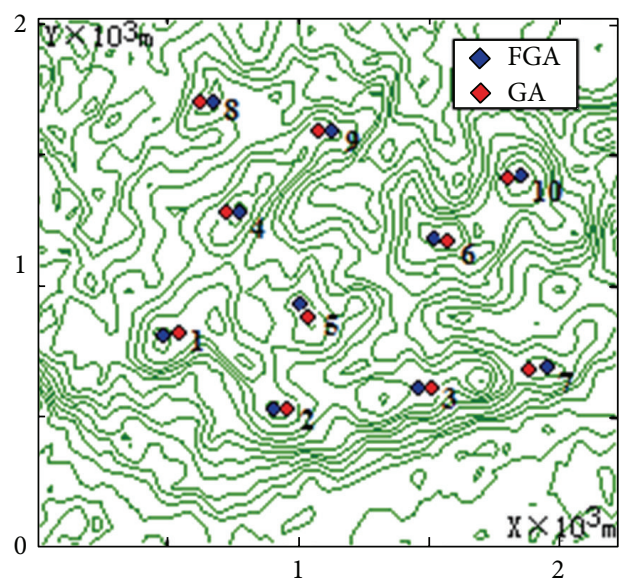

FIGURE 8: Final WTs layout using FGA and GA.

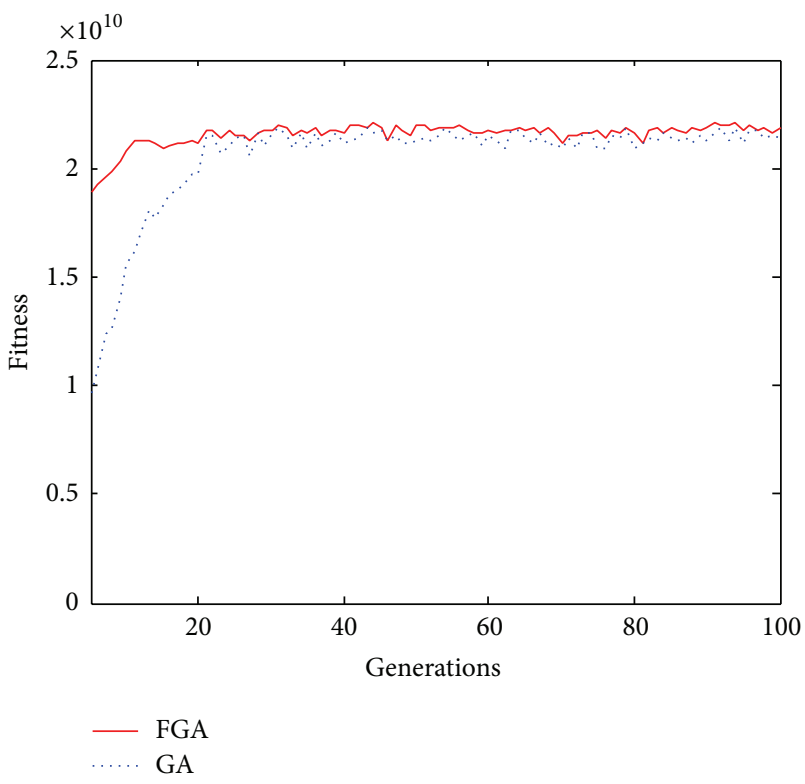

FIGURE 9: The optimal fitness values during the optimizations.

\section{Conflict of Interests}

The authors declare that there is no conflict of interests regarding the publication of this paper.

\section{Acknowledgments}

The project was supported by National Natural Science of China (Grant nos. 61104099 and 61374124), State Key Program of National Natural Science of China (Grant no. 61433004), Fundamental Research Funds for the Central Universities (Grant no. N130404008 and N130104001), and the R\&D Project of State Grid Corporation of China (DKYKJ[2012]001-2, GWKJ2013-005, and 2014-0-1-04244).

\section{References}

[1] Global Energy Research Council, "Global wind report-annual market update 2013," Tech. Rep. E2013-04-09, Global Energy Research Council, 2013, http://www.gwec.net/wp-content/ uploads/2014/04/GWEC-Global-Wind-Report 9-April-2014.pdf.

[2] S. F. Khahro, K. Tabbassum, A. M. Soomro et al., "Technoeconomical evaluation of wind energy potential and analysis of power generation from wind at Gharo, Sindh Pakistan," Renewable and Sustainable Energy Reviews, vol. 35, pp. 460-474, 2014.

[3] J. F. Manwell, J. G. McGowan, and A. L. Rogers, Wind Energy Explained Theory, Design and Application, John Wiley \& Sons, New York, NY, USA, 1st edition, 2003.

[4] A. Kusiak and Z. Song, "Design of wind farm layout for maximum wind energy capture," Renewable Energy, vol. 35, no. 3, pp. 685-694, 2010.

[5] S. Pookpunt and W. Ongsakul, "Optimal placement of wind turbines within wind farm using binary particle swarm optimization with time-varying acceleration coefficients," Renewable Energy, vol. 55, pp. 266-276, 2013.

[6] S. Brusca, R. Lanzafame, and M. Messina, "Wind turbine placement optimization by means of the Monte Carlo simulation method," Modelling and Simulation in Engineering, vol. 2014, Article ID 760934, 8 pages, 2014.

[7] G. Mosetti, C. Poloni, and B. Diviacco, "Optimization of wind turbine positioning in large wind farms by means of a genetic algorithm," Journal of Wind Engineering \& Industrial Aerodynamics, vol. 51, no. 1, pp. 105-116, 1994.

[8] I. Katic, J. Hojstrup, and N. Jensen, "A simple model for cluster efficiency," in Proceedings of the European wind energy Association Conference and Exhibition (EWEC'86), pp. 407-410, Rome, Italy, October 1986.

[9] G. Marmidis, S. Lazarou, and E. Pyrgioti, "Optimal placement of wind turbines in a wind park using Monte Carlo simulation," Renewable Energy, vol. 33, no. 7, pp. 1455-1460, 2008.

[10] Y. Eroĝlu and S. U. Seçkiner, "Design of wind farm layout using ant colony algorithm," Renewable Energy, vol. 44, pp. 53-62, 2012.

[11] Z. W. Geem and J. Hong, "Improved formulation for the optimization of wind turbine placement in a wind farm," Mathematical Problems in Engineering, vol. 2013, Article ID 481364, 5 pages, 2013.

[12] C. Wan, J. Wang, G. Yang, H. Gu, and X. Zhang, "Wind farm micro-siting by Gaussian particle swarm optimization with 
local search strategy," Renewable Energy, vol. 48, pp. 276-286, 2012.

[13] M. X. Song, K. Chen, Z. Y. He, and X. Zhang, “Optimization of wind farm micro-siting for complex terrain using greedy algorithm," Energy, vol. 67, pp. 454-459, 2014.

[14] N. Jensen, "A note on wind turbine interaction," Tech. Rep. RisM-2411, Risø National Laboratory, Roskilde, Denmark, 1983.

[15] R. J. Barthelmie, L. Folkerts, G. C. Larsen et al., "Comparison of wake model simulations with offshore wind turbine wake profiles measured by sodar," Journal of Atmospheric and Oceanic Technology, vol. 23, no. 7, pp. 888-901, 2006.

[16] W. Weng, P. A. Taylor, and J. L. Walmsley, "Guidelines for airflow over complex terrain: Model developments," Journal of Wind Engineering and Industrial Aerodynamics, vol. 86, no. 2-3, pp. 169-186, 2000.

[17] M. J. M. Stevens and P. T. Smulders, "The estimation of the parameters of the Weibull wind speed distribution for wind energy utilization purposes," Wind Engineering, vol. 3, no. 2, pp. 132-145, 1979.

[18] C. Ding, C. Zhang, and H. Liu, "Discussions on some issues in genetic algorithms," Information and Control, vol. 26, no. 1, pp. 40-47, 1979.

[19] K. W. Kim, M. Gen, and G. Yamazaki, "Hybrid genetic algorithm with fuzzy logic for resource-constrained project scheduling," Applied Soft Computing Journal, vol. 2, no. 3, pp. 174-188, 2003.

[20] H. G. Zhang and Y. B. Quan, "Modeling, identification, and control of a class of nonlinear systems," IEEE Transactions on Fuzzy Systems, vol. 9, no. 2, pp. 349-354, 2001.

[21] H. Zhang, S. Lun, and D. Liu, "Fuzzy $H_{\infty}$ filter design for a class of nonlinear discrete-time systems with multiple time delays," IEEE Transactions on Fuzzy Systems, vol. 15, no. 3, pp. 453-469, 2007.

[22] H. G. Zhang, J. Yang, and C.-Y. Su, "T-S fuzzy-model-based robust $\mathrm{H}$-infinity design for networked control systems with uncertainties," IEEE Transactions on Industrial Informatics, vol. 3, no. 4, pp. 289-301, 2007.

[23] H. Zhang and D. Liu, Fuzzy Modeling and Fuzzy Control, Birkhäauser, Boston, Mass, USA, 2006.

[24] H. G. Zhang, M. Li, J. Yang, and D. Yang, "Fuzzy model-based robust networked control for a class of nonlinear systems," IEEE Transactions on Systems, Man, and Cybernetics, Part A: Systems and Man, vol. 39, no. 2, pp. 437-447, 2009.

[25] J. Yang, Z. Q. Zeng, X. Huang, and Q. Y. Sun, "Research on wind power prediction based on distributed wind farms siting," Advanced Materials Research, vol. 805-806, pp. 312-315, 2013. 


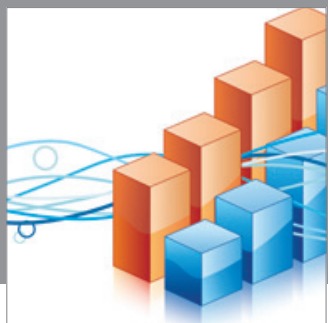

Advances in

Operations Research

mansans

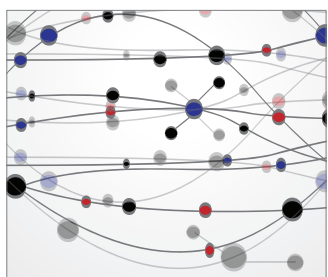

The Scientific World Journal
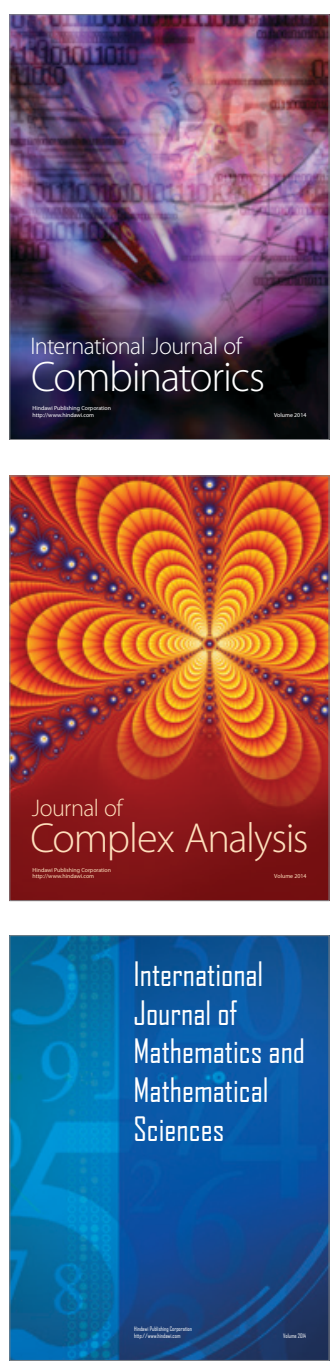
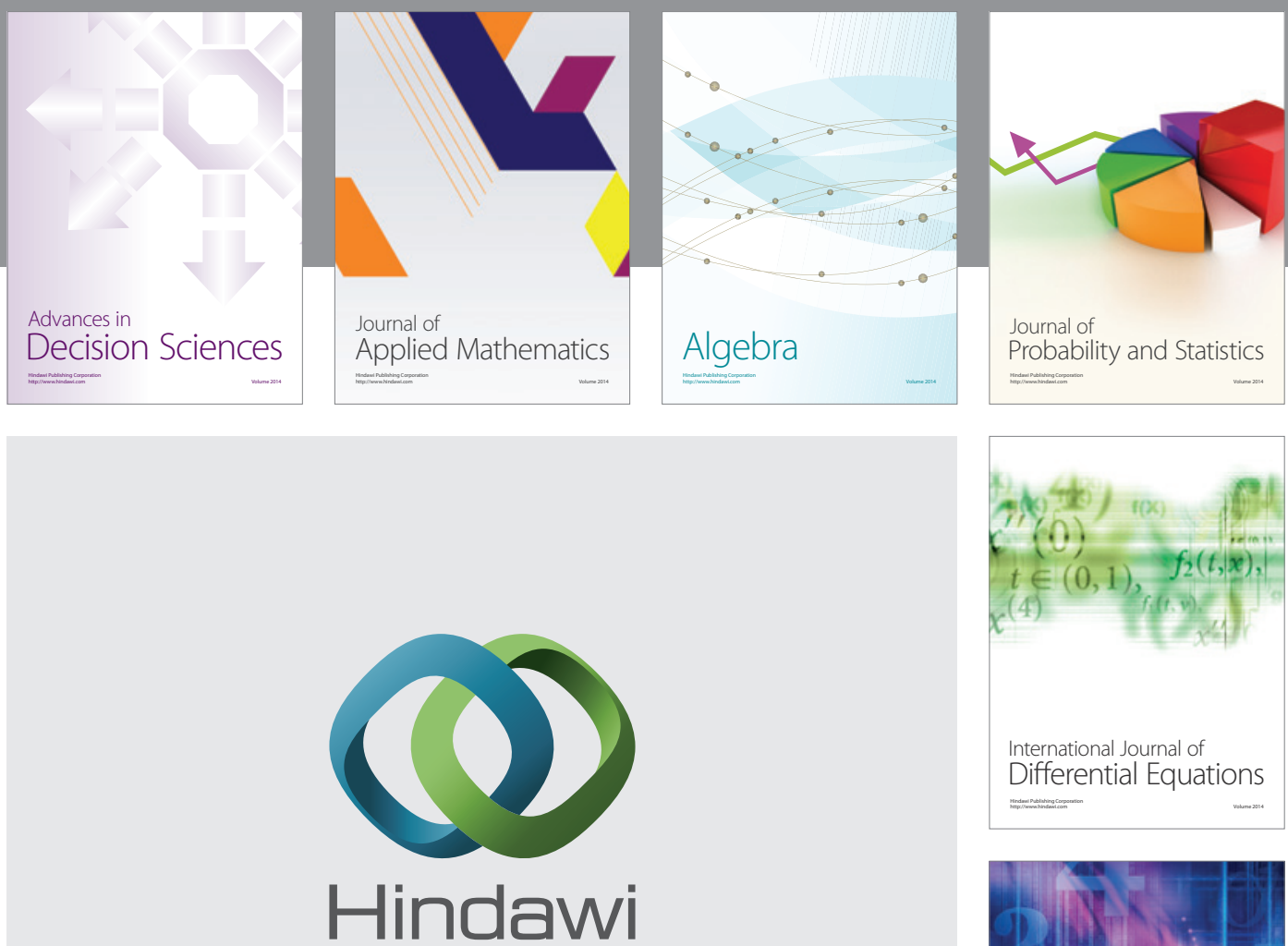

Submit your manuscripts at http://www.hindawi.com
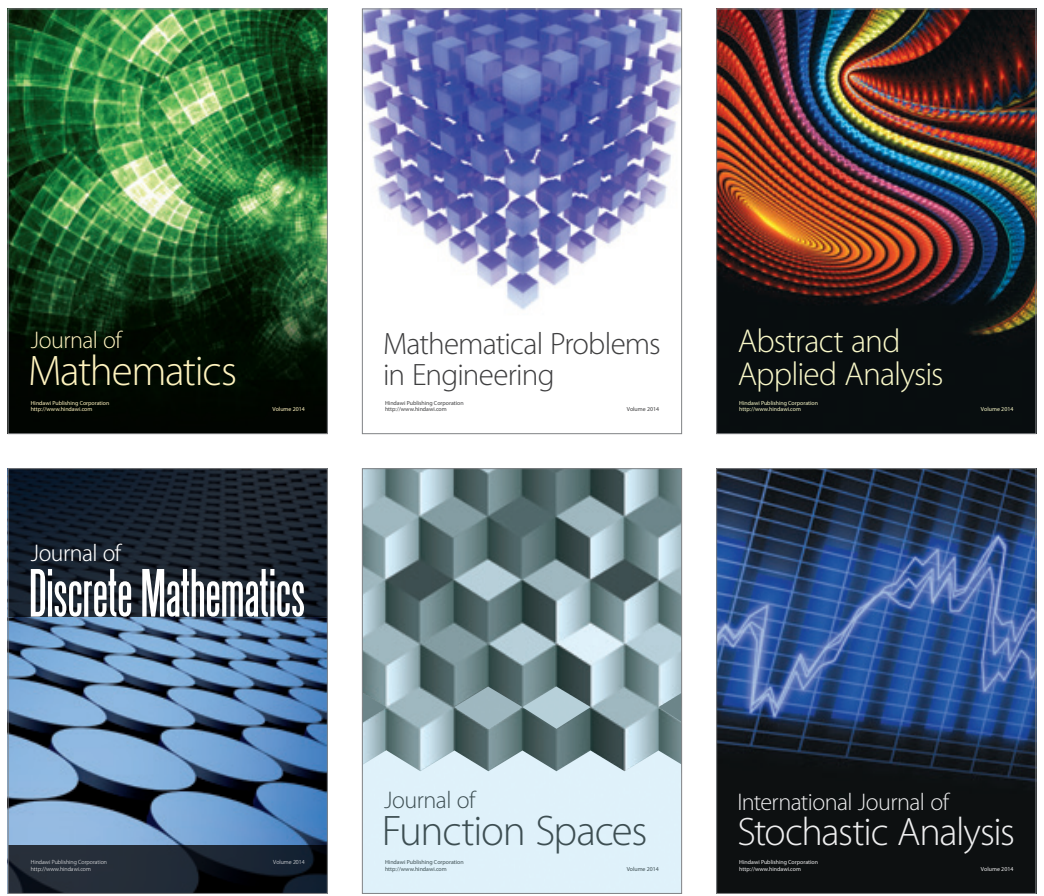

Journal of

Function Spaces

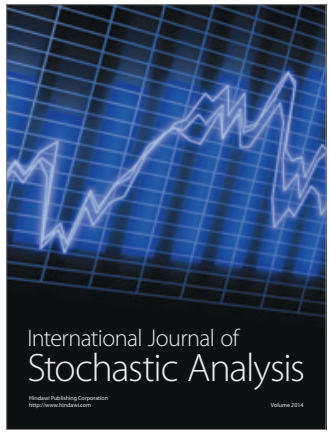

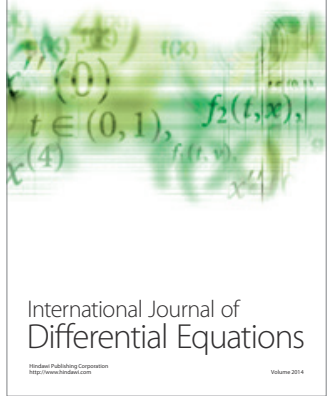
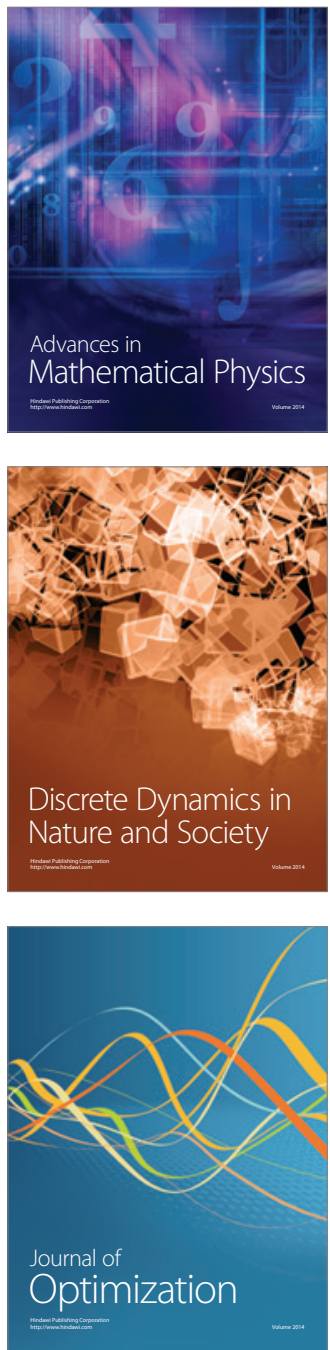